\title{
Familia Salvador. Biblioteca-Museo Salvador: breve bibliografía
}

\author{
Á. MELÉNDEZ-GALÁN \\ Servei de Biblioteca, Institut Botànic de Barcelona (IBB-CSIC-ICUB), pg. del Migdia, s/n, \\ ES-08038 Barcelona, España \\ E-mail: amelgal@gmail.com \\ Editor: N. Ibáñez
}

Recibido 6 octubre 2011; Aceptado 9 marzo 2012

\begin{abstract}
Salvador family. Salvador Library-Museum: Brief bibliography. - A brief bibliography about the Salvador family from Barcelona, its work and its "cabinet of curiosities" is presented here.

Key words: bibliography; Salvador family; Salvador Museum.

\section{Resumen}

Familia Salvador. Biblioteca-Museo Salvador: Breve Bibliografía.- Se aporta una breve bibliografía relativa a la familia Salvador de Barcelona, su trabajo y su «gabinete de curiosidades».

Palabras clave: bibliografía; familia Salvador; Museo Salvador.
\end{abstract}

\section{INTRODUCCIÓN}

Los Salvador (Fig. 1, A) fueron una familia de boticarios y naturalistas originarios de Calella, que vivieron en Barcelona entre los siglos XVII y XIX. Personas de gran curiosidad científica (apoyada por unos considerables recursos económicos), se relacionaron con algunos de los naturalistas de mayor relevancia a nivel internacional como Tournefort, Jussieu o Petiver. También fueron responsables de la creación de uno de los primeros jardines botánicos científicos y reunieron en su casa del carrer Ample esquina con Fusteria (en las cercanías de la actual Via Laietana), un auténtico museo (su «gabinete de curiosidades») compuesto por colecciones de animales conservados en alcohol o disecados (Fig. 1, C-D), fósiles y conchas, minerales, píldoras y compuestos químicos, semillas y muestras de madera $y$, lo que es más importante, el herbario y la biblioteca especializada en medicina, farmacia y ciencias naturales. Estas colecciones, recuperadas en 1937 de una masia del Penedès donde habían sido trasladadas a mediados del siglo XIX, se conservan actualmente en el Institut Botànic de Barcelona, donde parte de ellas se muestra al público en una exposición permanente.

Este trabajo no contiene un repertorio bibliográfico exhaustivo dada la ingente cantidad de obras que tratan directa o indirectamente sobre los Salvador; más bien, presenta una breve recopilación de bibliografía que, organizada por bloques temáticos, pretende servir como punto de partida o guía de lectura para todos aquellos que deseen iniciar investigaciones sobre la familia Salvador o 

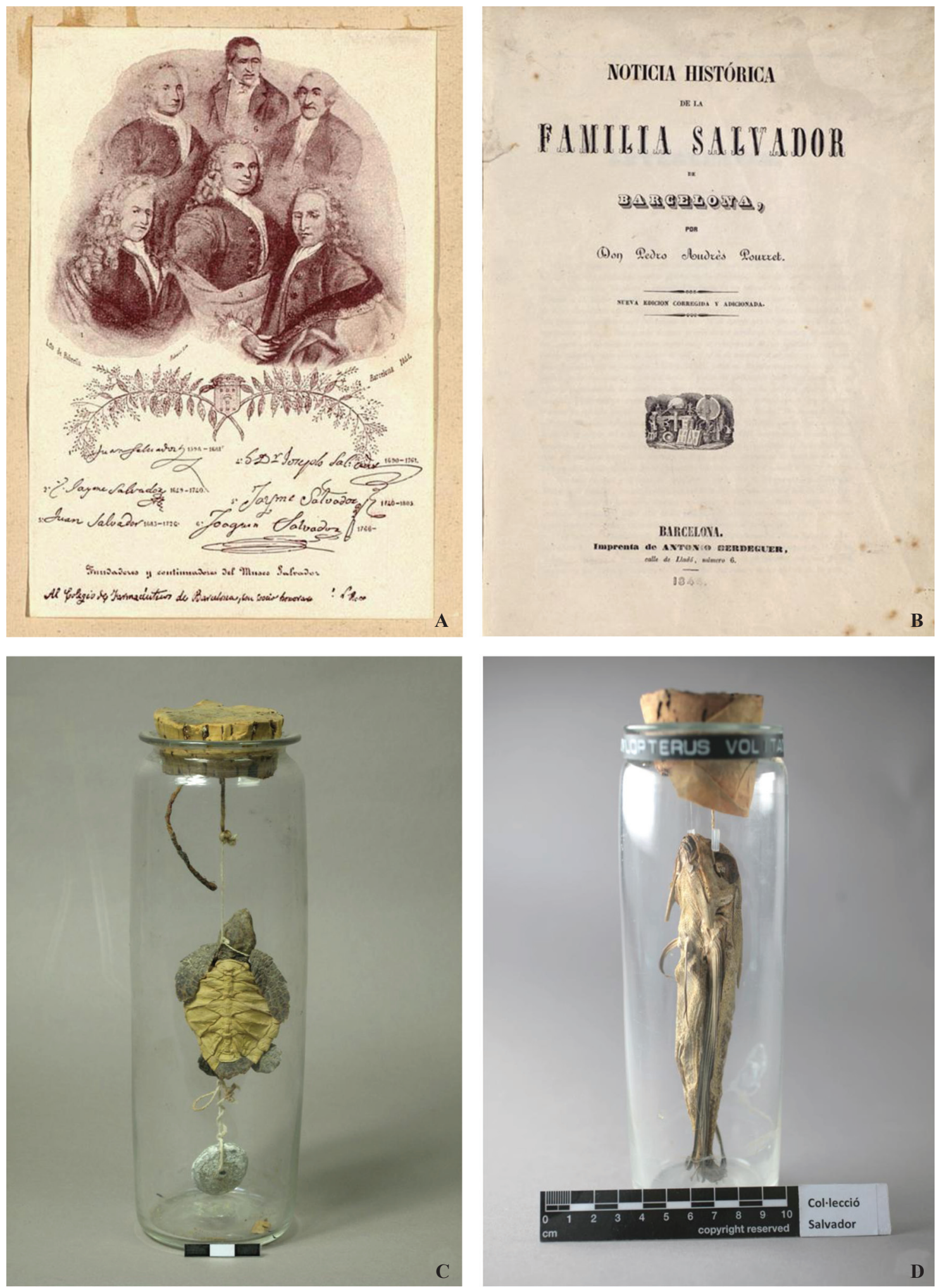

Figura 1. (A), los miembros más importantes de la familia Salvador. De izquierda a derecha y de arriba a abajo: Josep Salvador i Riera (1690-1761), Joaquim Salvador i Burgés (1766-ca. 1857), Jaume Salvador i Salvador (1740-1806), Joan Salvador i Boscà (1598-1681), Joan Salvador i Riera (1683-1725) y Jaume Salvador i Pedrol (1649-1740); (B), portada de la edición de 1844 de Noticia histórica de la familia Salvador de Barcelona de Pedro Andrés Pourret; (C) y (D), tarros de vidrio del Gabinete Salvador que contienen animales originalmente sumergidos en líquido conservante. En la imagen C puede observarse la pesa que mantenía el cuerpo del animal completamente sumergido. 
sus colecciones. La mayoría de los documentos citados a continuación pueden consultarse en la biblioteca del Instituto Botánico de Barcelona y algunos de ellos se encuentran disponibles a través de Internet en diversos repositorios de libre acceso. De esta recopilación cabe destacar la obra Noticia histórica de la familia Salvador de Barcelona, del botánico francés Pierre A. Pourret, a la cual hacen referencia la mayoría de los trabajos que tratan este tema y es un buen punto de partida para hacerse una idea general sobre la vida y obra de los miembros de la familia Salvador, con los que el autor tuvo una estrecha relación. De esta obra hay varias ediciones de distintos impresores correspondientes a los años 1796, 1828 y 1844. En el listado siguiente se cita la más reciente (Fig. 1, B) por ser la que se ha consultado.

\section{BIBLIOGRAFÍA}

\section{Gabinete Salvador}

[Bolòs, A. de?] 1942. El Museo Salvador: Nuevamente se instaló en el Instituto Botánico de Barcelona la colección Salvador. Chron. Bot. 7: 224.

Camarasa, J. M. 1988. El gabinete de curiosidades de la familia Salvador. Barc. Metròp. Mediterr. 9: 143-147.

Camarasa, J. M. 2004. De col·lecció privada a museu públic. El gabinet Salvador de 1'Institut Botànic de Barcelona. Afers. Fulls Recer. Pensam. 19: 721-727.

Camarasa, J. M. 2010. Les col·leccions d'història natural de Barcelona. Plecs Hist. Local 136: 5-7.

Fustagueras Fuster, J. 1858. Breve reseña de los archivos, bibliotecas, gabinetes, monetarios y museos. Imprenta de la Publicidad, de Antonio Flotats, Barcelona.

Gallegos, P. 2006. Un paseo por los museos cientificos de Barcelona. Un itinerario de cuatro siglos. Universitat Pompeu Fabra, Barcelona. Consultado el 4 de octubre de 2011, en http://www.upf. edu/pcstacademy/_docs/museos_bcn.pdf

Garcia Sastre, A. 1997. Els museus d'art de Barcelona: antecedents, gènesi $i$ desenvolupament fins l'any 1915. Publicacions de l'Abadia de Montserrat, Barcelona.

Madoz, P. 1850. Diccionario Geográfico-Estadistico-Histórico de España y sus posesiones de ultramar 3. Establecimiento Tipográfico de P.
Madoz y L. Sagasti, Madrid.

Masriera, A. 1978. El Museu de Geologia (Museu Martorell): Un segle d'història, 1878-1978. Museu de Geologia (Ajuntament de Barcelona), Barcelona.

Pardo Tomás, J. 2010. Escrito en la rebotica. Coleccionismo naturalista y prácticas de escritura en el gabinete de curiosidades de la familia Salvador. Barcelona, 1626-1857. Cult. Escr. Soc. 10: 17-52.

Pascual Bennasar, M. J. 2003. La historia natural de Bonaventura Serra i Ferragut (1728-1784), un ilustrado mallorquín. Servei d'Arxius i Biblioteques (Ajuntament de Palma), Palma.

Permanyer, L. 1993. El Gabinete de curiosidades de los Salvador. La Vanguardia Magazine, 24 de octubre de 1993: 46-50.

Pernau, G. 2004. Un Institut Botànic abocat a la ciutat. Barc. Metròp. Mediterr. 63: 8-12.

Vidal-Folch, I. 2006. El Gabinete Salvador. El País (Ed. Cataluña), 7 de enero de 2006: 2.

Zúñiga, R. 1994. El gabinete de curiosidades de la familia Salvador. Hist. 16, 213: 95-100.

\section{Correspondencia}

Camarasa, J. M. 2006. Joan Salvador - James Petiver: Xarxes epistolars. Afers. Fulls Recer. Pensam. 21: 37-49.

Camarasa, J. M. \& Amigó, J. J. 1993 Salvadorianae. I. La correspondència de Pere Barrère i Volar (Perpinyà 1690-1755) amb Josep Salvador i Riera conservada a la biblioteca Salvador de l'Institut Botànic de Barcelona. Collect. Bot. (Barcelona) 22: 73-104.

Camarasa, J. M. \& Ibáñez, N. 2007. Joan Salvador and James Petiver: a scientific correspondence (1706-1714) in time of war. Arch. Nat. Hist. 34: 140-173.

\section{Botánica}

Barceló Combis, F. 1881. Flora de las Islas Baleares, seguida de un diccionario de los nombres baleares, castellanos y botánicos de las plantas espontáneas y de las cultivadas. Establecimiento Tipográfico de Pedro José Gelabert, Palma.

Bolòs, A. de 1947. El Herbario Salvador. Collect. Bot. (Barcelona) 1: 1-8.

Bolòs, A. de 1947. Plantas montserratinas de Juan Salvador. Collect. Bot. (Barcelona) 1: 323-329. 
Camarasa, J. M. 1989. Botànica i botànics dels Països Catalans. Enciclopèdia Catalana, Barcelona.

Camarasa, J. M. 1989. Elements per a una història de la botànica i els botànics dels Països Catalans. Tesis Doctoral, Universitat de Barcelona, Barcelona.

Camarasa, J. M. 2000. Salvadorianae. III. Una descripció prelinneana de Silene niceensis All., deguda a Jaume Salvador i Pedrol, a l'edició de 1686 del Botanicum Monspeliense de Pèire Magnol. Collect. Bot. (Barcelona) 25: 245-253.

Camarasa, J. M., Camós, A. \& Sendra, C. 2007. La botànica i els sabers naturalístics i agronòmics. In: Vernet, J. \& Parés R. (Eds.), La ciència en la història dels Països Catalans 2. Del naixement de la ciència moderna a la Il.lustració. Institut d'Estudis Catalans \& Universitat de València, València: 555-602.

Camarasa, J. M. \& Català, J. I. 2007. Els nostres naturalistes 1 (Monografies Mètode, 2). Universitat de València, València.

Colmeiro Penido, M. 1846. Catálogo metódico de plantas observadas en Cataluña, particularmente en las inmediaciones de Barcelona [...] y seguido de la nomenclatura catalana de las plantas traducida al idioma castellano y al botánico [...]. Viuda e hijos de Don Antonio Callejas, Madrid. Colmeiro Penido, M. 1858. La botánica y los botánicos de la península Hispano-lusitana. Estudios biográficos y bibliográficos. Imprenta de Rivadeneyra, Madrid.

Garganta, M. de 1938. L'Herbari Salvador. Rev. Catalunya 85: 564-568.

Ibáñez, N. 2006. Estudis sobre cinc herbaris històrics de l'Institut Botànic de Barcelona. Tesis Doctoral, Universitat de Barcelona, Barcelona.

Ibáñez, N., Montserrat, J. M. \& Soriano, I. 2004. Plantas cultivadas en el Herbario Salvador (Siglos XVII-XVIII). Póster presentadado en el 2nd World Botanic Gardens Congress (Barcelona, 17-22 de abril de 2004).

Ibáñez, N., Montserrat, J. M., Soriano, I. \& Camarasa J. M. 2006. Plant material exchanged between James Petiver (ca. 1663-1718) and Joan Salvador i Riera (1683-1725). I. The Balearic Plants conserved in the BC-Salvador and BMSloane Herbaria. Notes \& Rec. Roy. Soc. London 60: 241-248.

Montserrat, J. M. \& Ibáñez, N. 2006. Les plantes ornamentals i la botànica. In: García, E., Rivero, M., Montserrat, J. M. \& Ibáñez, N. (Eds.), Jardins, jardineria i botànica. Barcelona 1700. Ajuntament de Barcelona, Barcelona: 113-163. Rodríguez Femenías, J. J. 1865-1868. Catálogo razonado de las plantas vasculares de Menorca. Tip. de Fábregues hermanos, Mahón.

Romo, A. M. 1991. The Salvador Herbarium at the Institut Botànic de Barcelona (BC): An 18th Century herbarium of Mediterranean plants. O. P. T. I. M. A. Newslett. 25-29: 48-49.

Romo, A. M. 1995. Un herbario prelinneano en el Institut Botànic de Barcelona: el herbario Salvador (finales del siglo XVII y principios del XVIII). Bol. Asoc. Herb. Ibero-Macaronés. 0: 4-5.

Salvador Riera, J. 1972. Viatge d'Espanya i Portugal (1716-1717). Edicions 62, Barcelona.

Valderas, J. M. 2000. Francisco Vélez de Arciniega en la polémica de la coloquíntida. Asclepio 52: 7-36.

Vila, J. 1960. Notas Históricas. El Jardín Botánico de San Juan Despí, su emplazamiento. Rev. R. Acad. Farm. Barcelona 61: 51-58.

\section{Biblioteca}

Camarasa, J. M. 1994. La Biblioteca i les col·leccions Salvador de l'Institut Botànic de Barcelona: una font per a l'estudi de la història natural a Catalunya de mitjan segle XVII a mitjan segle XIX. In: Camarasa, J. M., Mielgo, H. \& Roca, A. (Eds.), Actes de les I Trobades d'Història de la Ciència i de la Tècnica: Trobades Científiques de la Mediterrània. Institut d'Estudis Catalans, Barcelona: 157-166.

García Ramiro, A. M. \& Plaza-Navas, M. A. 2008. Catalogación retrospectiva del fondo bibliográfico del Museo Salvador. Collect. Bot. (Barcelona) 27: 113-116.

Montserrat, J. M. \& Pardo Tomás, J. (Eds.) 2008. Catàleg de la Biblioteca Salvador, Institut Botànic de Barcelona. Consejo Superior de Investigaciones Científicas, Madrid.

Olmi, G. 2008. La Biblioteca dels Salvador en la república de les lletres: amb motiu de la presentació del catàleg de la Biblioteca Salvador de l'Institut Botànic de Barcelona, 5 de juny de 2008. Delegació del CSIC a Catalunya, Barcelona. 


\section{Fósiles}

Abad, A. 1997. La colección de Petrefactos del Museo de los Salvador de Barcelona. Siglos XVII, XVIII y primera mitad del XIX. Batalleria 7: $57-73$.

\section{Familia Salvador}

Boada, C. 1997. Nissaga d'apotecaris. Avui, 12 de enero de 1997: 30.

Bolòs, A. de 1946. Los botánicos de la familia Salvador y el Instituto Botánico de Barcelona. Divulg. Hist. 4: 195-199.

Bolòs, A. de 1959. Nuevos datos para la historia de la Familia Salvador. Por el Muy Iltre. Dr. Antonio de Bolós Vayreda-Discurso de contestación por el Muy Iltre. Dr. Taurino Mariano Losa España (Discursos de Recepción, 3). Real Academia de Farmacia de Barcelona, Barcelona.

Bolòs, A. de 1964. Tournefort et Jaume Salvador. Adansonia 3: 3-4.

Camarasa, J. M. 1993. Salvadorianae, II. Les Jussieu et les Salvador. Deux familles de naturalistes au début du XVIIIe siècle. In: Layssus, Y. (Ed.), Les naturalistes français en Amerique du Sud, XVIe-XIXe siècles. Actes du 118 e Congrès national des sociétés historiques et scientifiques. Comité des Travaux Historiques et Scientifiques, Paris: 69-102.

Devesa Alcaraz, J. A. \& Viera Benítez, M. C. 2001. Viajes de un botánico sajón por la Península Ibérica (H. M. Willkomm, 1821-1895). Servicio de Publicaciones de la Universidad de Extremadura, Cáceres.

Fluvià, A. de 2000. Informe sobre la noblesa de la familia Salvador. Paratge. Quad. Estud. Geneal. Her. Sigillogr. Vexillol. 11: 101-112.

Folch Jou, G. 1955. Juan Salvador y Boscá. Bol. Soc. Esp. Hist. Farm. 6: 117.
Folch Jou, G. 1956. Jaime Salvador y Pedrol. Bol. Soc. Esp. Hist. Farm. 7: 25-26.

Fontcuberta, L. 1983. Joan Salvador i Riera (16831725): Tricentenari d'un científic català massa oblidat. Progrés. Butll. barri Collblanc-La Torrassa 2: 12.

Fradera, J. M. 1993. Ciència i negoci amb rerafons colonial al segle XIX català (set vinyetes i un epíleg). L'Avenç 172: 30-57.

García Cárcel, R. 1985. Historia de Cataluña, siglos XVI-XVII 1. Los caracteres originales de la historia de Cataluña. Ariel, Barcelona.

Martí Escayol, M. A. 2001. Catalunya dins la xarxa científica de la il·lustració. John Polus Lecaan: medicina i botànica a Barcelona durant la Guerra de Successió. Manuscrits 19: 175-194.

Pourret, P. A. 1844. Noticia histórica de la familia Salvador de Barcelona. Nueva edición corregida $y$ adicionada. Imprenta de Antonio Berdeguer, Barcelona.

Roldán Guerrero, R. 1956. Juan Salvador y Riera. Bol. Soc. Esp. Hist. Farm. 7: 115-117.

Torras Ribé, J. M. 1990. La comarca de l'Anoia en les descripcions de viatgers dels segles moderns (1494-1834). Misc. Aqualatensia 6: 133-171.

Vidal Campederrós, N. 1855. Elogio fúnebre de D. José Salvador y Soler, abogado y socio de la Academia de Ciencias Naturales y Artes de Barcelona, y miembro de varias otras corporaciones. Leído á [sic] sobre dicha Academia de Ciencias Naturales y Artes en la sesión pública, celebrada en 21 de Octubre de 1855. Imprenta Nueva. Barcelona.

\section{Pesca}

Lleonart, J. \& Camarasa, J. M. 1987. La pesca a Catalunya el 1772: segons un manuscrit de Joan Salvador i Riera. Museu Marítim (Diputació de Barcelona), Barcelona. 